\title{
Cardiovascular CT in Cyanotic Congenital Heart Disease
}

\author{
Anjali Chelliah ${ }^{1,2} \cdot$ Amee M. Shah ${ }^{3} \cdot$ Kanwal M. Farooqi $^{3} \cdot$ Andrew J. Einstein $^{4} \cdot$ B. Kelly Han ${ }^{5}$
}

Published online: 7 June 2019

(C) The Author(s) 2019

\begin{abstract}
Purpose of Review To discuss the role of cardiovascular computed tomography (CT) in diagnosing and managing a spectrum of cyanotic congenital heart defects before and after repair and to review practical imaging considerations in patients with specific cyanotic lesions.

Recent Findings As CT technology has rapidly advanced and radiation doses have dramatically decreased, cardiovascular CT has provided a lower-risk, high-quality alternative to diagnostic cardiac catheterization or cardiac magnetic resonance imaging (MRI) and has been increasingly utilized in nearly every type of cyanotic congenital heart defect.

Summary Cardiovascular CT can provide rapid, high-resolution images to aid in preoperative planning and postoperative surveillance of cyanotic heart disease. To optimize each study, protocols should be carefully tailored to specific defects and to each patient's unique clinical and hemodynamic considerations.
\end{abstract}

Keywords Cardiac computed tomography $\cdot$ Pediatrics $\cdot$ Congenital heart disease $\cdot$ Cyanotic congenital heart disease $\cdot$ Single ventricle disease

\section{Introduction}

Congenital heart disease (CHD) - structural abnormalities of the heart and cardiac vessels that occur in fetal developmentis the most common type of birth defect, affecting 8 to 9 of every 1000 children [1]. Approximately $25 \%$ of these children have cardiac lesions characterized as cyanotic $[2,3]$. In cyanotic forms of CHD, a net right-to-left cardiac shunt allows desaturated systemic venous blood to bypass the lungs and enter the systemic arterial circulation. This shunt leads to

This article is part of the Topical Collection on Cardiac Computed Tomography

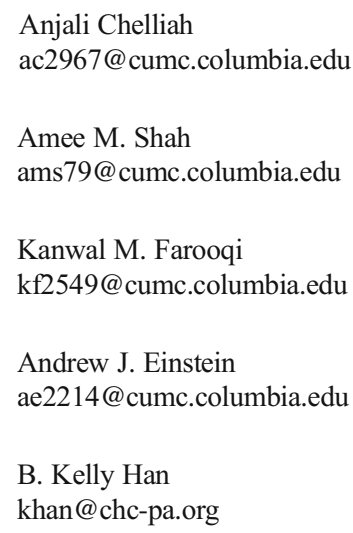

decreased arterial saturations and an increased deoxygenated hemoglobin level that manifests as central cyanosis when it exceeds $5 \mathrm{~g} / \mathrm{dl}$. A broad spectrum of complex defects, including tricuspid or pulmonary atresia and total anomalous pulmonary venous return (TAPVR), can cause right-to-left shunts. In defects such as tetralogy of Fallot (TOF) or pulmonary atresia with intact ventricular septum (PA/IVS), right ventricular outflow tract obstruction leads to decreased pulmonary blood flow and intracardiac shunting. Mixing of pulmonary and systemic circulations, as seen in truncus
Division of Pediatric Cardiology, Goryeb Children's Hospital, Morristown Medical Center, Morristown, NJ, USA

2 Division of Pediatric Cardiology, Columbia University Irving Medical Center, New York, NY, USA

3 Division of Pediatric Cardiology, Columbia University Irving Medical Center and Morgan-Stanley Children's Hospital of New York-Presbyterian, New York, NY, USA

4 Division of Cardiology, Department of Medicine, and Department of Radiology, Columbia University Irving Medical Center and New York-Presbyterian Hospital, New York, NY, USA

5 The Children's Heart Clinic at The Children's Hospitals and Clinics of Minnesota and The Minneapolis Heart Institute and Foundation, Minneapolis, MN, USA 
arteriosus and d-transposition of the great arteries (d-TGA), can also cause a net right-to-left shunt. Defects with left-sided cardiac obstruction, like hypoplastic left heart syndrome, typically cause low cardiac output and shock but can sometimes present with desaturation from right-to-left shunting across a patent ductus arteriosus to provide systemic blood flow [4]. Cyanosis can also present in older unrepaired patients with longstanding systemic to pulmonary shunting, such as an unrestrictive ventricular septal defect, in whom pulmonary arterial hypertension has developed and reversed the shunt to right-to-left, known as Eisenmenger syndrome.

While a growing percentage of CHD patients are diagnosed prenatally by obstetric ultrasound or echocardiogram, in the USA, about two-thirds are discovered postnatally [5]. Postnatally diagnosed cyanotic CHD often presents acutely in the newborn period or may be identified by routine pulse oximetry screening in the newborn nursery [6-8]. It typically requires surgical repair, palliation, or catheter-based intervention in the neonatal period or early infancy to alleviate desaturation. Therefore, even when a defect is prenatally diagnosed, the precise anatomic details that affect management must be determined quickly after birth. Congenital cardiologists often have a limited time window in the first hours to days of life to image the heart and decide upon the timing of intervention, procedure type, and surgical approach. These decisions depend in part on the infinitely many anatomic variations found in complex CHD, affecting visceroatrial situs, ventriculoarterial connections, great vessel anatomy and size, coronary artery arrangement, the presence, location, and size of septal defects, and systemic and pulmonary venous anatomy.

As management techniques are refined and CHD survival improves, the patient population has grown rapidly to approximately 2.4 million in the USA, including 300,000 with complex defects that include repaired or palliated cyanotic CHD [9]. The growing need for lifelong surveillance after repair has increased the demand for congenital cardiac imaging, which serves as a cornerstone of complex CHD management to assess residual lesions, monitor function, identify complications, and plan subsequent interventions.

Transthoracic echocardiography is the primary tool used to assess cardiac anatomy and function. It is readily available, relatively inexpensive, and poses little risk, even to unstable patients. However, despite typically good acoustic windows in infants and children, it can be difficult to determine details of small, often sub-centimeter structures and extracardiac vessels and reproducibly assess ventricular size and function. Echocardiographic windows are more limited in adult CHD patients, especially those with extensive sternal scarring after multiple interventions. Diagnostic cardiac catheterizationbased angiography, historically a mainstay of congenital cardiac imaging, provides superior spatial and temporal resolution [10]. However, invasive angiography is increasingly used primarily for hemodynamic evaluation rather than anatomic definition due to its risks. Catheterization is invasive, requires central vascular access, requires sedation or anesthesia in young patients, and exposes patients to radiation. Thus, the use of invasive catheterization to define complex congenital heart defect anatomy has declined with advances in noninvasive imaging [11-13].

In the last few decades, cardiovascular MRI has emerged as a high-quality, "one-stop shop" modality for imaging CHD. It permits three-dimensional (3D) visualization of cardiac structures and precise measurement of ventricular size and function without radiation. It is considered the gold standard for assessing the right ventricle (RV) in repaired tetralogy of Fallot [14]. In patients with cyanotic CHD, MRI flow sequences can quantify valve regurgitation and assess the ratio of pulmonary to systemic blood flow, differential pulmonary blood flow, and collateral flow [15]. Myocardial parametric mapping by MRI can detect micro-level fibrosis and edema [16]. Gadolinium contrast-enhanced images can also create magnetic resonance (MR) angiograms, identify focal myocardial fibrosis, and assess perfusion during rest and stress [17, 18]. However, these studies are very time-consuming. Even with advances in real-time and free-breathing imaging, congenital cardiac MRIs can last up to an hour or more and require patients to remain motionless and comply with repeated breath holds $[15,19]$. Children and some older patients with developmental delays therefore often require general anesthesia, raising concerns about potentially deleterious effects on developing brains in children whose cyanotic lesions are already associated with neurocognitive delays [20-22]. Anesthesia induction also carries a higher risk of cardiac arrest and mortality from arrest in children with CHD, particularly in cyanotic single ventricle patients who are unrepaired or partially palliated $[23,24]$. The use of MRI is also limited in patients with implanted devices that may be ferromagnetic or cause significant imaging artifact; this applies to many repaired cyanotic CHD patients with coils, stents, pacemakers, and defibrillators. Cardiac MRI poses additional technical challenges in neonates and infants, whose fast heart rates and small structures limit spatial resolution, signal-to-noise ratio, and the accuracy of volume and flow measurements [15]. Finally, recent data emerging on the risks of gadolinium deposition in the central nervous system and other organs have prompted an FDA advisory on gadolinium contrast, making MRI a less benign imaging option $[25,26]$.

\section{Benefits of Cardiovascular CT in Cyanotic CHD}

As the risk-benefit ratio of cardiac MRI has shifted, cardiac $\mathrm{CT}$ has played a rapidly expanding role in managing complex $\mathrm{CHD}$ and planning surgical and catheter-based interventions $[12,27]$. Technical advances have driven much of this 
increase [28]. Older scanners were limited by long scan times and low temporal resolution, but arguably, the most significant barrier to their use was high radiation doses.

Radiation exposure is of concern in all children but is particularly a matter of concern for those with cyanotic CHD [29]. They may undergo numerous chest x-rays, catheterizations, nuclear stress tests, and lung perfusion scans. A recent study from a large pediatric cardiac center documented cumulative lifetime radiation effective doses of up $77 \mathrm{mSv}$ among their CHD patients [30]. Another group reported that single ventricle patients received on average $10 \mathrm{mSv}$ per year during the first 3-4 years of life [31]. Older CT scanners, using a retrospectively electrocardiogram (ECG)-gated scan mode, have been reported to expose CHD patients to effective doses of up to $28 \mathrm{mSv}$ per cardiac scan [32]. Since then, innovations such as prospective ECG gating, ECG-controlled tube current modulation, high-pitch helical scanning, lower tube potentials, wider detector coverage, and iterative reconstruction techniques have dramatically lowered radiation exposure $[33,34]$. Today, sub-milliSievert scans are achievable in many children [35]. However, effective doses still vary widely, and recently published pediatric cardiac-specific dose-length product conversion or " $k$-"factors suggest that $k$-factors, and therefore effective cardiac radiation doses, in children are considerably higher than previously believed $[32,36]$. In an effort to minimize radiation doses and standardize imaging parameters across pediatric centers, the Image Gently alliance's "Have-a-Heart" campaign recently published radiation management guidelines for pediatric cardiovascular CT [32]. Several large congenital cardiac centers have also recently established an international congenital cardiovascular CT registry to establish best practice guidelines for radiation exposure and anesthesia use in CHD patients [37].

Newer CT scanner platforms also allow rapid image acquisition that decreases the need for sedation or anesthesia. With dual-source scan technology and wide detector coverage, studies can be obtained in a single heartbeat without requiring a breath hold for most indications. General anesthesia and paralysis are not necessary in most patients $[35,36]$. While cardiac MRI in critically ill and often unstable patients poses significant risks because of study length, need for sedation, the need to change MRI-incompatible ventilators and lines, and difficulties with closely monitoring patients inside the scanner, cardiac CT mitigates these risks. CT can be done in patients on ventilators, or even on extracorporeal membrane oxygenation (ECMO) or ventricular assist device support, as long as the patient can be transported safely to the scanner $[38,39]$. The same study can also evaluate for pulmonary artery embolism or thrombosis, which in particular has been reported in patients with Eisenmenger physiology, and image the lungs and airways, which are often abnormal in CHD patients [40, 41]. These factors make cardiac CT an attractive option for imaging cyanotic $\mathrm{CHD}$ patients.
In recent years, cardiac $\mathrm{CT}$ has also helped to advance the use of patient-specific 3D printing and augmented or virtual reality modeling for use in planning complex intracardiac repairs and other CHD interventions [42]. Digital 3D modeling and printing aid users in "mentally rotating" imaging data, which allows them to better understand complex spatial relationships within the heart and extracardiac structures [43]. Any volumetric Digital Imaging and Communications in Medicine (DICOM) dataset, including contrast-enhanced and non-contrast MR angiograms and 3D echo data, can be used to generate models, but $\mathrm{CT}$ is the most common source of 3D cardiac models and is considered the easiest to use [42, 44]. It confers submillimeter isotropic spatial resolution, and its bright contrast facilitates the process of segmenting or identifying anatomic structures to be printed. Figure 1 demonstrates a cardiac CT angiogram performed in a 1-day-old cyanotic neonate with prenatally diagnosed complex double outlet RV with an inlet ventricular septal defect, d-malposed great arteries with the aorta rightward of the pulmonary artery, and an aortic coarctation. The CT, performed with an effective radiation dose of about $0.3 \mathrm{mSV}$, was used delineate the relationships of the ventricles, VSD, and outflows and to create a detailed, hollowed-out scale model of the patient's heart. Although the infant was initially anticipated to undergo a staged repair over the first months of life to connect each ventricle to its appropriate outflow, the CT and 3D model permitted the surgeon to plan a complex intracardiac baffle repair that was successfully performed in a single operation at 2 weeks of age [45].

\section{The Role of Cardiovascular CT in Managing Specific Cyanotic Heart Defects}

Cardiovascular CT has been utilized to diagnose and manage a broad spectrum of cyanotic cardiac defects by delineating anatomic details, assessing cardiac relationships to extracardiac structures, and identifying post-intervention complications [12, 27]. In the following section, we will review its uses as they pertain to specific cyanotic defects.

\section{Tetralogy of Fallot and Other Right Ventricular Outflow Tract Obstructive Lesions}

TOF is the most common form of cyanotic CHD, accounting for 5\% of congenital heart defects [46]. In TOF, anterior deviation of the conal portion of the ventricular septum obstructs blood flow through the RV outflow tract and directs desaturated blood though a ventricular septal defect into the systemic circulation. RV outflow tract obstruction falls along a spectrum ranging from minimal subpulmonary stenosis to pulmonary atresia or, in its most severe form, absent main and branch pulmonary arteries with multiple aortopulmonary 
Fig. 1 a Segmentation of a cardiac $\mathrm{CT}$ in a 1-day-old neonate with complex double outlet right ventricle, d-malposed great vessels, and an aortic coarctation, in preparation for rapid prototyping. b 3D-printed and digital cardiac models (lateral walls removed to show intracardiac anatomy)

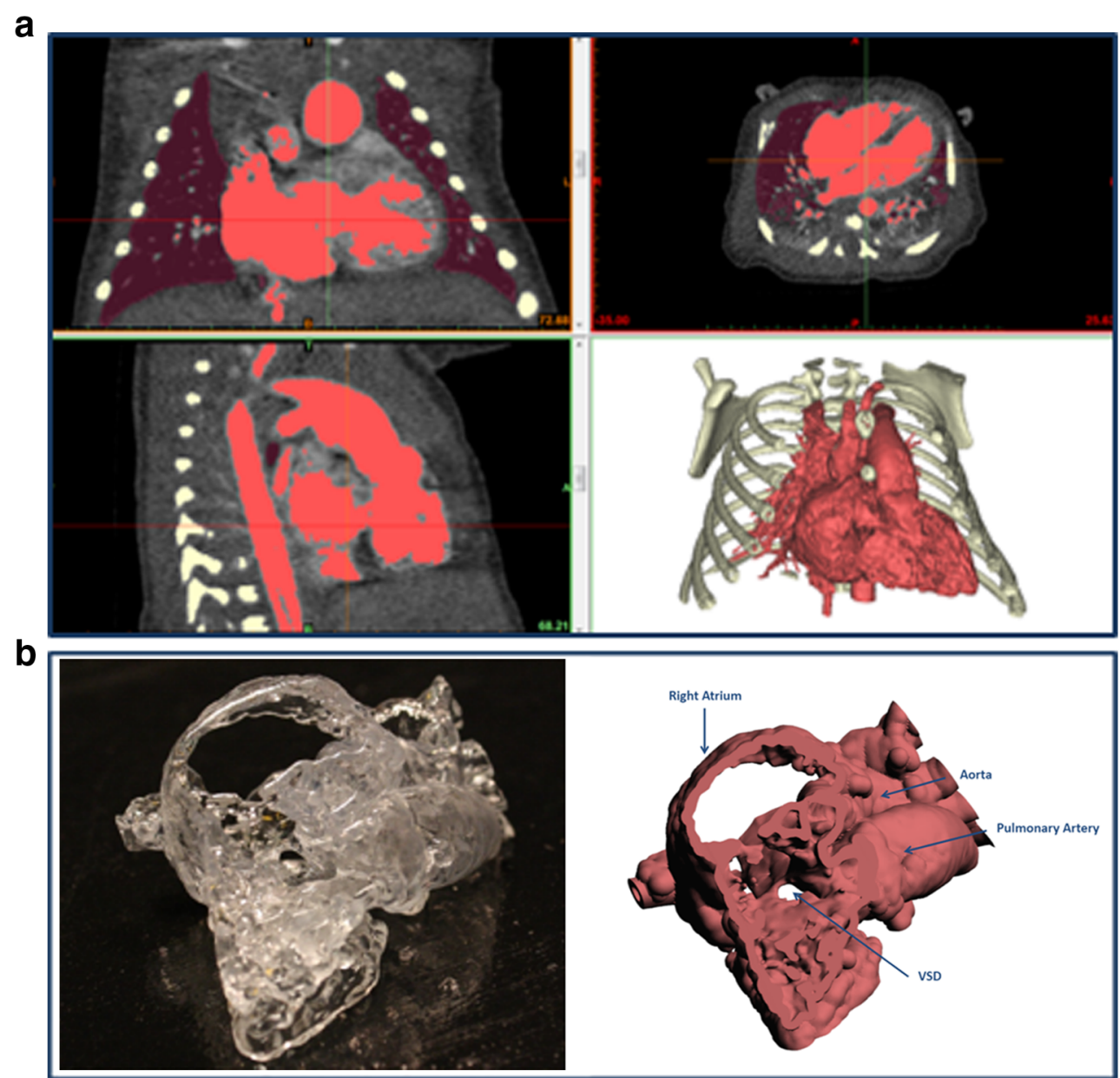

collaterals (MAPCAs) arising from the aortic arch to feed the lungs. Preoperatively, most TOF anatomy can be determined by echocardiogram. However, an estimated $5 \%$ to $12 \%$ of these patients have coronary artery abnormalities, such as an accessory left anterior descending coronary arising from the right coronary and crossing the pulmonary outflow tract, which can preclude a full neonatal repair [47-49]. Echo may be unable to definitively assess coronary artery anatomy, but $\mathrm{CT}$ has been shown to identify coronary anomalies in young children with unrepaired TOF with $97 \%$ to $100 \%$ sensitivity and specificity $[50,51]$.

In TOF with MAPCAs, the tortuous network of very small collateral vessels is rarely seen well on echocardiogram or even on cardiac MRI. Traditional management strategy involved obtaining a diagnostic cardiac catheterization under general anesthesia within a few days of birth. However, the excellent spatial resolution of cardiovascular CT makes it an optimal modality to visualize even distal aortopulmonary collateral anatomy [52-54]. In many institutions, CT has become the first-line imaging modality to delineate aortopulmonary collateral anatomy upon birth or initial patient presentation. $\mathrm{CT}$ can determine the presence and anatomy of native pulmonary arteries, courses of the MAPCAs, the lung segments they supply, and the relationship of each collateral to the airways and esophagus (Fig. 2). Catheterization may therefore be delayed until the patient is older, decreasing risk of vessel injury relative to during the newborn period. When preoperative catheterization is performed, usually at a few months of age, CT images guide the procedure, which focuses on identifying aortopulmonary collateral stenosis and dual sources of pulmonary blood flow. Fewer angiograms can be performed, limiting radiation and contrast exposure. Some centers have also started to use CT angiograms to create 3D digital and printed models, which can be sterilized and placed on the surgical field as an intraoperative guide $[55,56]$.

In TOF patients who undergo staged repair and are initially palliated with aortopulmonary shunts, CT is often obtained prior to full repair to evaluate shunt patency and assess for branch pulmonary artery stenoses or aneurysms. In repaired TOF patients, $\mathrm{CT}$ can assess for right ventricular to pulmonary artery (RV to PA) conduit patency and calcification, pseudoaneurysms, and pulmonary artery stenosis $[57,58]$. In patients with contraindications to cardiac MRI, functional CT has been used to assess RV volume and function. Most reports demonstrate relatively 
Fig. 2 Cardiac CT volume renderings in a 2-day-old boy with prenatally diagnosed TOF with pulmonary atresia and MAPCAs, demonstrating aortopulmonary collaterals (asterisk) arising from the descending thoracic aorta and confluent but severely hypoplastic native branch pulmonary arteries (short arrow). Long arrow denotes the connection of an aortopulmonary collateral to the left pulmonary artery

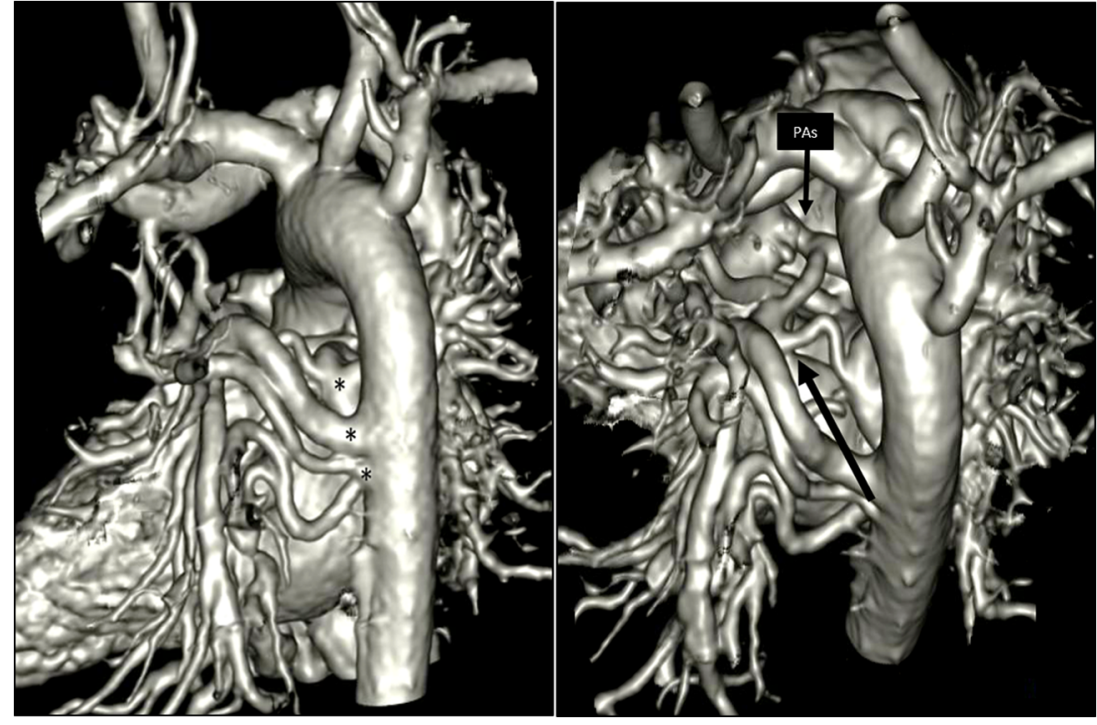

equivalent volume assessment between $\mathrm{CT}$ and MRI if a CT scanner with appropriate temporal resolution is used, but mild overestimation of RV end-diastolic volumes has also been reported $[59,60]$.

CT can also demonstrate the proximity of the outflow tract to the coronary arteries (Fig. 3), which recent TOF management guidelines for adult congenital CHD patients recommend assessing in all patients to help determine candidacy for percutaneous pulmonary valve replacement [61]. CT was also recently reported to identify thrombus and endocarditis, not demonstrated on echocardiogram, as a cause of percutaneous pulmonary valve obstruction [62]. Before repeat surgical intervention, CT can identify the relationship of the RV outflow tract to the sternum to minimize the risk of dissecting an adherent or calcified pulmonary artery or conduit [27].

Several other cyanotic heart defects also involve pulmonary outflow obstruction or are repaired with RV to PA conduits, including variants of double outlet right ventricle with RV outflow tract obstruction that are repaired with a Rastelli procedure. Cardiac CT can play a similar role in these patients' surveillance and management. In patients with PA/IVS, in which the pulmonary valve is completely obstructed and the $\mathrm{RV}$ is often smaller and hypertensive, cardiac CT has been used in newborns to identify commonly associated coronary abnormalities such as coronary stenoses and RV to coronary artery fistulae $[59,60]$.
Fig. 3 Nineteen-year-old man with double outlet RV and pulmonary stenosis status postRastelli operation, with RV to PA conduit stenosis. Cardiac CT identified the LMCA coursing just inferior to the conduit, a contraindication to RV outflow tract stenting

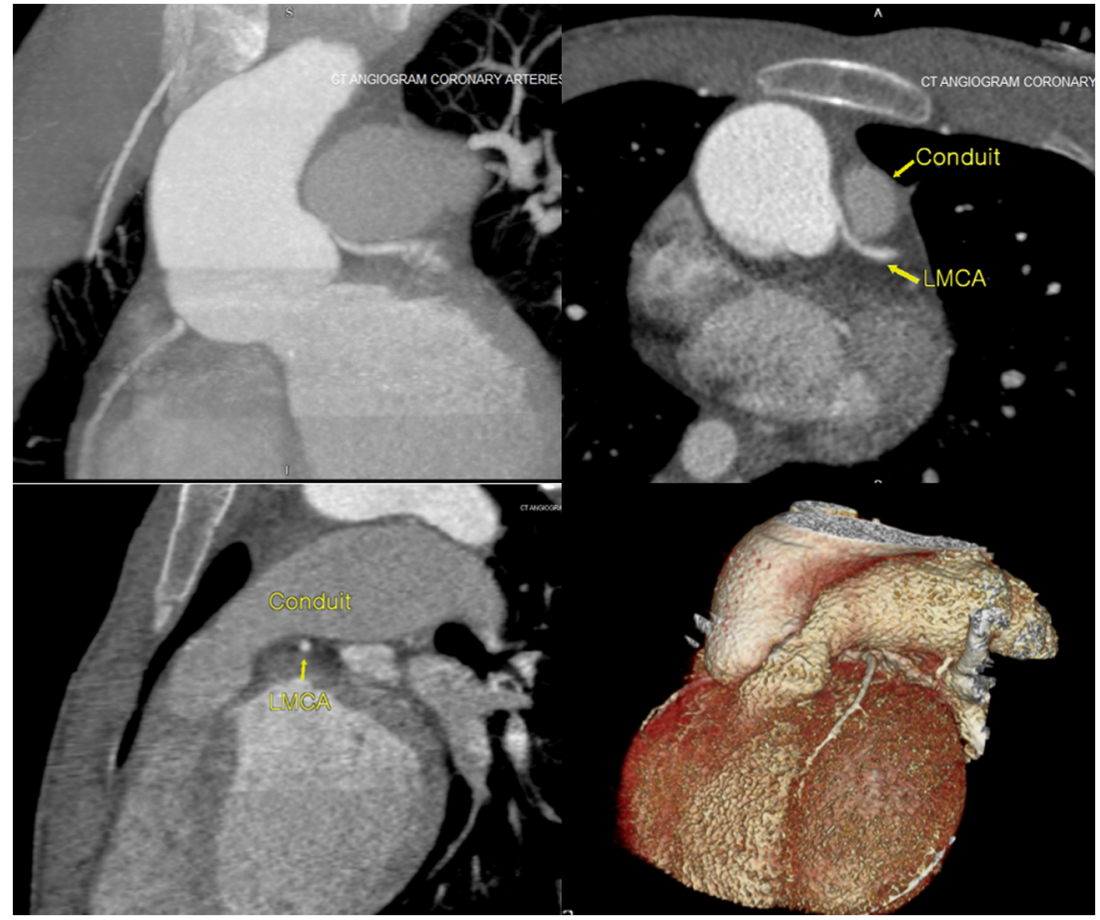




\section{Transposition of the Great Arteries}

Transposition of the great arteries refers to several types of defects with ventriculoarterial discordance, but the most common is "simple" d-TGA without a ventricular septal defect and with unobstructed outflow tracts [63]. In d-TGA, which represents $3 \%$ to $5 \%$ of congenital heart defects, patients typically undergo an arterial switch operation (ASO) within the first week of life [1]. Preoperative anatomy is usually determined by echocardiogram, but dual-source cardiovascular CT in neonates has been reported to identify abnormal coronary artery arrangements, which occur in up to one-third of d-TGA patients, with higher sensitivity than echocardiography [64-66]. Postoperatively, cardiovascular CT is often used to identify complications such as coronary artery stenosis after reimplantation, which is reported in $8 \%$ to $10 \%$ of patients who have undergone ASO [67-70]. CT is also commonly used to assess for main and branch pulmonary artery obstruction after surgical LeCompte maneuver (Fig. 4) as well as neoaortic root dilation [70]. While CT perfusion imaging may be useful to evaluate for myocardial perfusion defects resulting from coronary artery obstruction, its use has been reported only in conjunction with positron emission tomography (PET) in this population [71]. In patients with more complex forms of dTGA with a ventricular septal defect and pulmonary outflow obstruction, who undergo a Rastelli operation with RV to PA conduit placement or a Nikaidoh operation with translocation of the aorta and occasional reimplantation of the right coronary artery, $\mathrm{CT}$ is again a useful option to assess residual lesions and guide reintervention (Fig. 5) [72].

Older patients with d-TGA who were repaired in the 1970s and 1980s typically underwent a Mustard or Senning atrial switch operation, in which systemic and pulmonary venous drainage to the atria was redirected towards the appropriate outflows. Atrial switch complications typically include baffle leaks or obstruction and dysfunction of the systemic RV that remains connected to the aorta [73]. Cardiac CT can be used to assess baffle obstruction, patency of intrabaffle stents, and

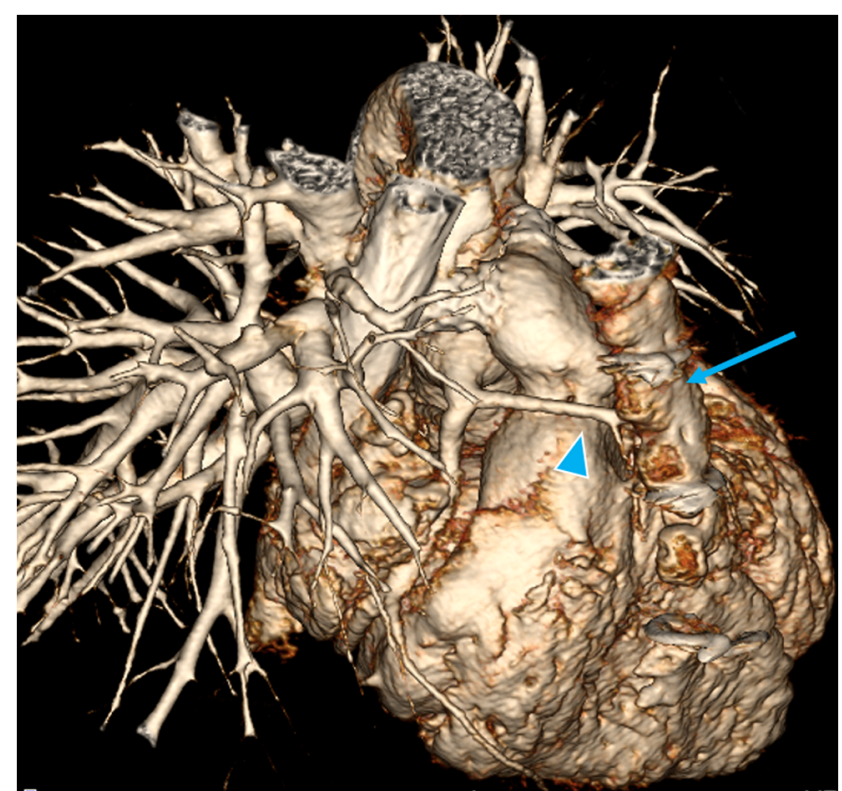

Fig. 5 Eleven-year-old girl with d-TGA status post-arterial switch operation. Volume rendering of cardiac CT obtained for preoperative planning prior to surgical reintervention demonstrates the left anterior descending coronary artery (blue triangle) arising from the right neoaortic sinus and coursing between the sternum (blue arrow) and main pulmonary artery, posing risk of coronary injury during repeat sternotomy

ventricular size and function [74]. While the Mustard and Senning are typically no longer performed for d-TGA, the atrial switch has reemerged in congenitally corrected or levo-transposition of the great arteries (1-TGA), either in conjunction with the arterial switch as part of the double switch procedure to address discordance at both the atrioventricular and ventriculoarterial levels or along with a Rastelli procedure in patients with l-TGA, VSD, and pulmonary stenosis (Fig. 6) $[75,76]$. Many patients with atrial switch repair of d-TGA, who are prone to arrhythmias, and 1-TGA, in which atrioventricular block is common, have also undergone pacemaker or defibrillator placement. CT is therefore a more optimal
Fig. 4 Two-month-old boy with d-TGA status post arterial switch operation. a Maximum intensity projection (MIP) demonstrating stenosis of the proximal branch pulmonary arteries after LeCompte maneuver. b Volume rendering of the branch pulmonary arteries viewed from the cranial perspective
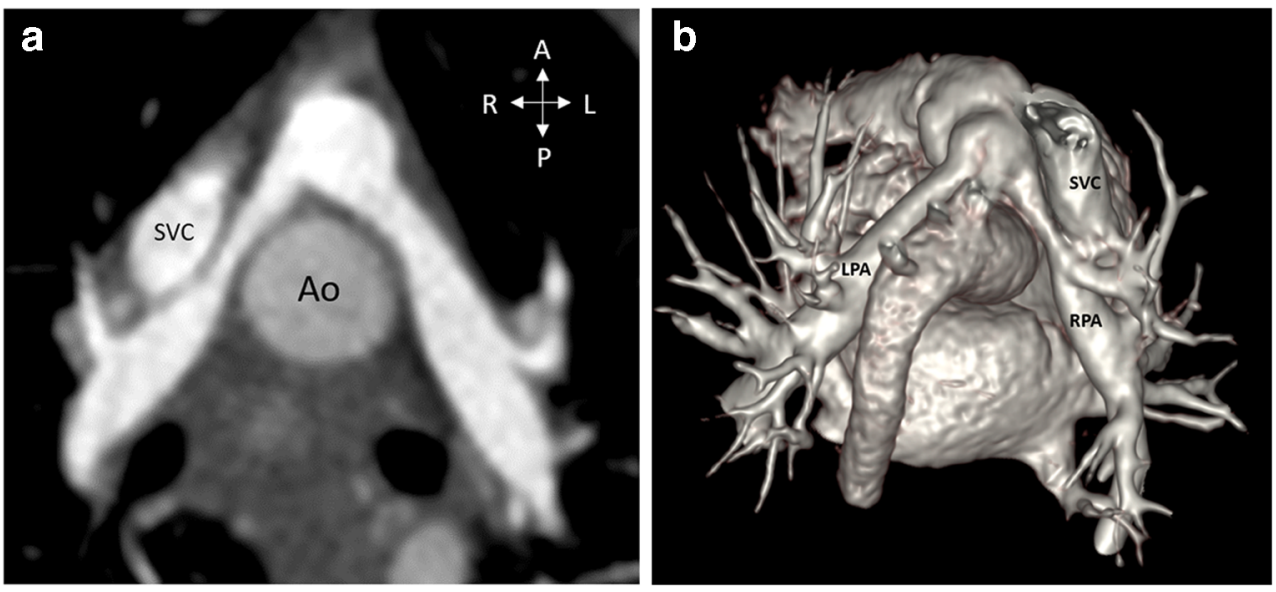


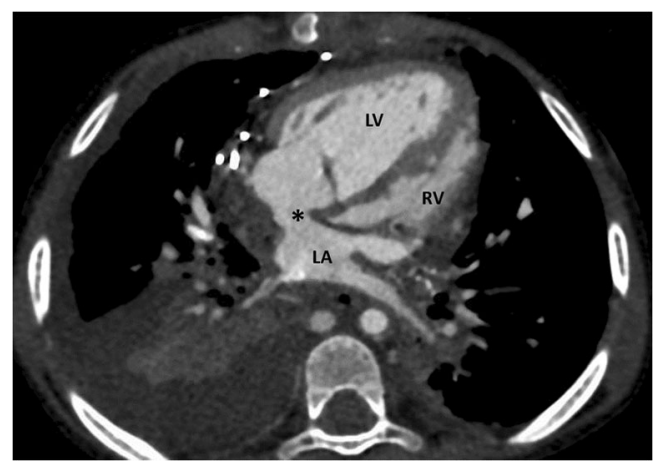

Fig. 6 Two-year-old girl with 1-TGA, ventricular septal defect, and a hypoplastic left-sided RV, status post-hemi-Mustard, Rastelli, and bidirectional Glenn operations. Asterisk denotes the pathway connecting the pulmonary veins to the right-sided LV

modality than MRI to visualize anatomy and function and guide $\mathrm{EP}$ interventions in these patients [73, 74].

\section{Truncus Arteriosus}

Truncus arteriosus is a relatively rare- $0.5 \%$ of $\mathrm{CHD}-$ defect in which the pulmonary and aortic outflow tracts fail to separate during fetal development and remain as a single outflow, allowing mixing of systemic and pulmonary venous blood [1]. CT is rarely used preoperatively for this lesion, which is usually well delineated on echocardiogram. However, preoperative CT has been reported to better demonstrate the branch pulmonary arteries and rule out associated findings such as an interrupted aortic arch $[77,78]$. Postoperatively, CT can assess sequelae related to both the left heart, which has an abnormal truncal valve that is often eventually replaced with a mechanical valve, and right heart, in which an RV to PA conduit is placed or direct connection is created. Figure 7 demonstrates the left ventricular outflow tract of a 15-year-old patient with truncus arteriosus and recent infective endocarditis. Echocardiogram could not clearly visualize the truncal valve region due to mechanical valve artifact, but cardiovascular CT demonstrated a large diverticulum of the truncal root that compressed his RV to PA conduit.

\section{Total Anomalous Pulmonary Venous Return}

In TAPVR, which makes up $1 \%$ to $2 \%$ of $\mathrm{CHD}$, the pulmonary veins connect abnormally to various sites in the systemic veins, including the superior vena cava (supracardiac type), inferior vena cava (infracardiac), coronary sinus, or a combination of sites. Cyanosis is often worsened by obstruction of the anomalous connection, particularly in the infracardiac type, which may cause pulmonary congestion and severe hemodynamic instability. Cardiovascular CT can be rapidly performed even in critically ill patients and can preoperatively identify the course of the pulmonary veins, drainage sites, and areas
Fig. 7 Cardiac CT MIPs (a, b) and volume rendering $(\mathbf{c})$ in a $15-$ year-old boy with truncus arteriosus status post-RV-PA conduit placement and mechanical truncal (aortic) valve replacement complicated by infective endocarditis. A diverticulum was seen on $\mathrm{CT}$ arising from the left ventricular outflow tract coursing between the ascending aorta and RV-PA conduit and compressing the conduit. A small area of hypoattenuation (asterisk) suggestive of thrombus is also seen within the diverticulum
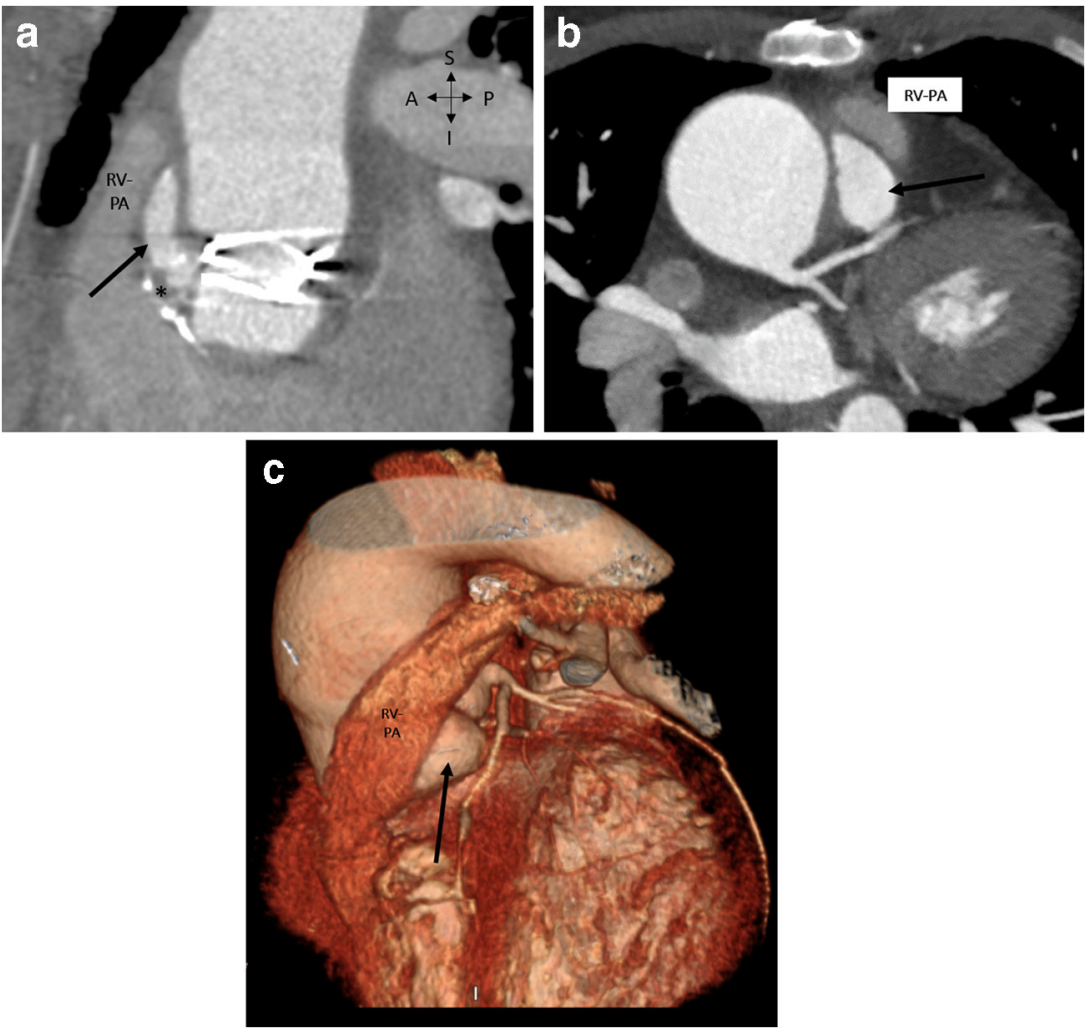
of obstruction [79-82]. Post-repair, CT is a preferred modality to assess recurrent pulmonary venous obstruction, which can occur in up to $18 \%$ of patients (Fig. 8) [83]. Cardiovascular CT is also useful for pulmonary vein imaging in patients with pulmonary venous abnormalities in the setting of more complex defects, such as heterotaxy syndrome or single ventricle disease, and can clearly delineate the sites of venous drainage and mechanisms of obstruction (Fig. 9).

\section{Single Ventricle Disease and Heterotaxy Syndrome}

Single ventricle or functionally single ventricle defects cause cyanosis secondary to right-to-left shunting, parallel systemic and pulmonary circulations, and in some cases pulmonary outflow obstruction. These defects include tricuspid atresia and other forms of hypoplastic right heart syndrome, hypoplastic left heart syndrome, double inlet left ventricle (LV), double outlet RV, unbalanced atrioventricular canal defects, and severe forms of Ebstein anomaly. Most of these are palliated over the first 3 to 4 years of life by connecting the systemic venous return directly to the pulmonary arteries and utilizing the single ventricle as the systemic ventricle, pumping through an unobstructed native aorta or an augmented "neoaorta" constructed in part from the pulmonary artery. As many of these defects are associated with abnormalities in the great vessels and systemic and pulmonary venous connections, echocardiography does not always adequately determine complex anatomic details prior to palliation. Cardiovascular CT is commonly used in these neonates for preoperative planning and is often performed using a nonsedated "feed and wrap" method of swaddling and allowing the patient to fall asleep after eating [84-86]. Some single

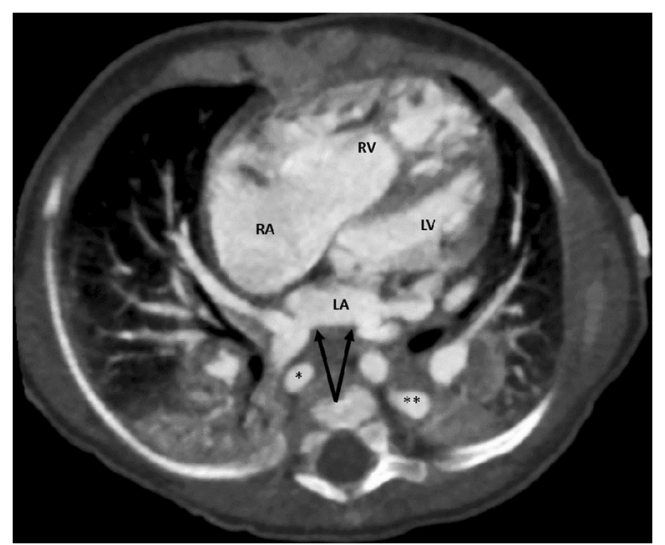

Fig. 8 Three-month-old girl with repaired supracardiac TAPVR and discrete narrowing of the right lower pulmonary venous confluence and left lower pulmonary vein as they enter the left atrium. The left upper pulmonary vein (not shown) is occluded and drains instead via the azygous (one asterisk) and hemiazygous (two asterisks) veins into the superior vena cava. The right heart is dilated due to pressure and volume overload ventricle disease occurs in the setting of heterotaxy syndrome, in which the laterality of structures within the thorax and abdomen is arranged abnormally, with highly variable duplication of left- or right-sided structures known as isomerism. In these patients, CT can assess not only intracardiac and extracardiac abnormalities but can also identify bilateral left or right atrial appendages and assess the situs of the lungs and abdominal viscera [87].

Postoperatively, CT has been used to assess shunt patency, pulmonary artery stenosis, aortic arch obstruction, and venovenous or aortopulmonary collateral vessels [57, 88]. It has also been used in pre-Glenn and pre-Fontan procedural planning, in some cases as a noninvasive alternative to diagnostic cardiac catheterization. In a series of 32 single ventricle patients, half of whom underwent cardiovascular CT and half of whom underwent diagnostic cardiac catheterization prior to second stage palliation, CT had no diagnostic discrepancies compared to surgical findings. Patients were exposed to much less radiation than those who underwent catheterization (effective dose $1.1 \mathrm{mSv}$ compared to $14 \mathrm{mSv}$ ), received less than half the contrast of the catheterized cohort, and had only one adverse event - increased cyanosis - compared to eight events in the catheterized patients [89]. After palliation, CT can evaluate Glenn and Fontan pathways for obstruction or thrombus (Fig. 10) [90]. Due to mixing of contrastopacified and non-opacified blood as the contrast enters the pulmonary arteries from the SVC, injection protocols should be optimized to achieve heterogeneous contrast within the vessels and avoid false-positive diagnosis of thrombus $[57,91]$. A number of solutions have been proposed, including simultaneous arm and leg injection and lower extremity injection, but many sources agree that delayed scanning ranging from 60 to $180 \mathrm{~s}$ after injection, which may be guided by a timing bolus, yields homogeneous contrast opacification throughout the heart and cavopulmonary pathways [92, 93].

\section{Practical Considerations for Cardiovascular CT in Cyanotic CHD}

Performing cardiovascular CT in patients with cyanotic cardiac defects, who span the spectrum of neonates to adults and may be unrepaired, palliated, or fully repaired, requires careful individualization of the exam to the patient, the physiology of the defect, and the clinical question being addressed. Detailed guidelines addressing scan protocols and radiation dose reduction in pediatric and congenital heart disease have been published elsewhere, but we will briefly review a few considerations pertinent to cyanotic CHD patients [28, 29, 32, 94-96]. 

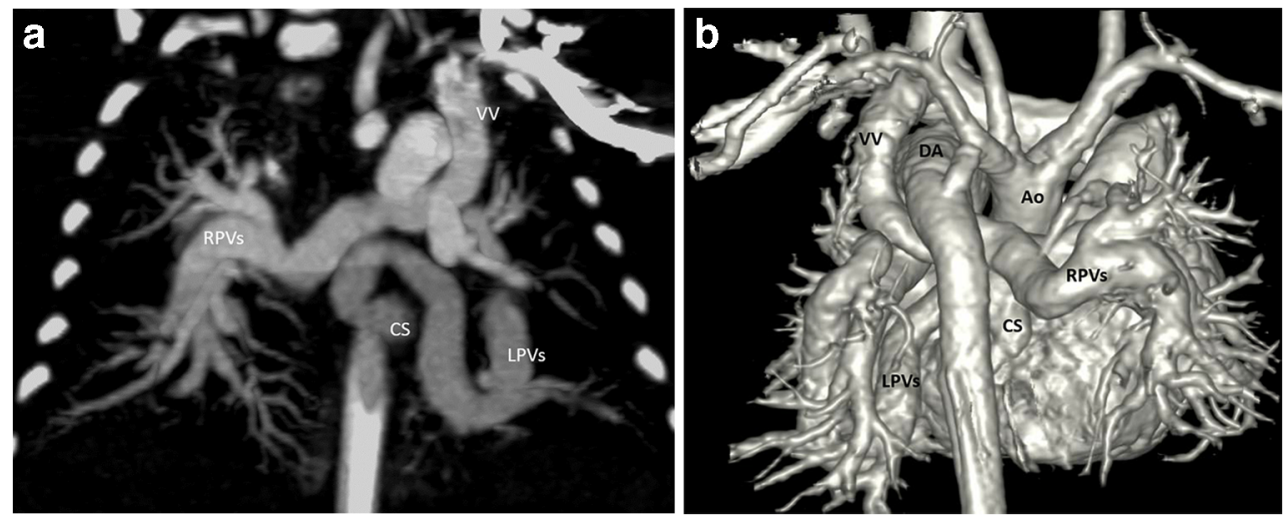

Fig. 9 Coronal MIP (a) and volume rendering from the posterior view (b) obtained in a neonate with hypoplastic left heart syndrome to delineate pulmonary venous drainage. The right-sided pulmonary veins (RPVs) cross the midline to a left-sided vertical vein (VV) entering the

innominate vein. The left-sided pulmonary veins (LPVs) drain to a tortuous channel that enters the coronary sinus $(\mathrm{CS})$. DA = ductus arteriosus, Ao $=$ aorta

\section{Sedation}

The need for sedation can vary depending on patient characteristics, CT scanner speed, and exam goals. For many CTs, developmentally appropriate older children, typically over the age of 4 years, can remain motionless in the scanner and by 6 or 7 years can cooperate with breath hold instructions [95]. Of note, children with cyanotic CHD are well-documented to have a high incidence of neurodevelopmental abnormalities and genetic syndromes, which should be determined before the study [22, 97]. Infants under 6 months can often utilize the "feed and wrap" method outlined above. However, particularly in studies focusing more on extracardiac structures such as the aortic arch or pulmonary arteries or veins, whose images are less motion-sensitive, we find that even toddlers and preschool-aged children may be scanned without sedation if tightly wrapped or placed in a vacuum immobilizer device. Child life therapists are often involved for support, and a parent wearing appropriate shielding can remain in the room

when possible during the scan. For coronary artery imaging in younger children, general anesthesia with suspended respiration is often required to minimize motion artifact. Although this scenario is not ideal, these cases are often performed as a lower-risk alternative to cardiac catheterization, which would expose these patients to general anesthesia, an invasive procedure, and increased radiation.

Older 64-slice scanners that use prospectively ECGgated "step-and-shoot" technology, in which sequential axial scans are obtained while the table moves along the $z$-axis with each gantry rotation, may require up to $10 \mathrm{~s}$ to scan the entire chest [94]. Resulting images may therefore demonstrate "stair-step" artifact due to patient motion between sequential acquisitions. Patients being scanned with this technology are more likely than those imaged with high-pitch or wide coverage scanners to require sedation, particularly when images are being used for 3D modeling or printing. In ICU patients who are already intubated and sedated, our team often requests paralysis and suspended
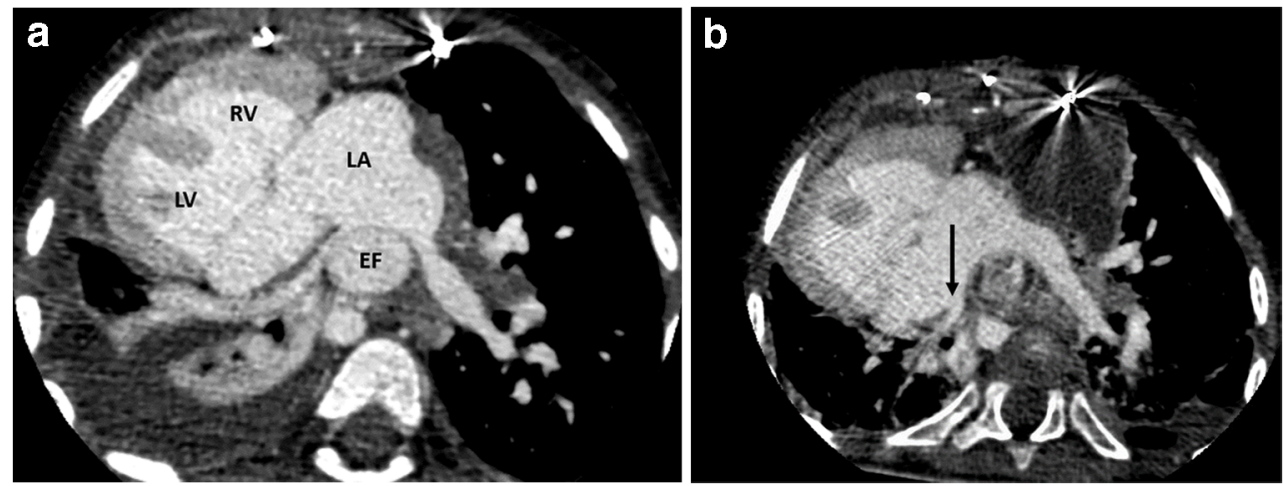

Fig. 10 Complex heterotaxy with dextrocardia, congenitally corrected transposition of the great arteries with 1-looped ventricles (RV is anterior and leftward of the LV), and pulmonary valve atresia. a Cardiac CT obtained post-Fontan palliation demonstrates compression of the right-sided pulmonary veins (arrow) by an extracardiac Fontan

conduit coursing posterior to the left-sided atrium. b Subsequent CT post-pulmonary venoplasty demonstrates persistent right pulmonary vein narrowing (arrow) as well as focal hypoattenuation within the Fontan conduit suggestive of thrombus 
respiration to optimize study quality without imposing significant additional clinical risk.

\section{Intravenous Access and Contrast}

Vascular access may be difficult in complex CHD patients who have undergone multiple hospital admissions and interventions since birth. Intravenous (IV) placement should be performed with attention to each patient's systemic and pulmonary venous anatomy and any known occlusions or collateral vessels to ensure appropriate opacification of the heart [57]. Typically, the speed of power injection is limited by IV gauge, which may range from less than $1 \mathrm{ml} / \mathrm{s}$ using a 24gauge IV in a neonate to $5 \mathrm{ml} / \mathrm{s}$ using an 18-gauge IV in a teenager or adult. As image contrast is generally optimized when scanning at lower tube potentials $(\mathrm{kVs})$ that generate $\mathrm{x}$-rays with energies closer to the k-edge of iodine, it is not essential to push contrast particularly rapidly and risk extravasation [98]. Contrast dose may be determined more by the desired duration of contrast opacification at a given injection rate than by patient weight but typically ranges from 1.5 to $2 \mathrm{ml} / \mathrm{kg}$.

Patients with any form of cyanotic CHD are known to have a high prevalence - up to $50 \%$ by adulthood - of chronic kidney disease resulting from insults related to polycythemic hyperviscosity, diminished cardiac function, nephrotoxic medication exposure, and cardiopulmonary bypass runs $[99,100]$. Therefore, care should be taken to pre-hydrate if needed, avoid concomitant nephrotoxic medications, and use low-osmolar or iso-osmolar contrast such as iohexol or iodixanol [94].

As with all procedures in cyanotic CHD patients, even small air bubbles should be carefully avoided during injection to avoid crossing right-to-left shunts. When injecting contrast in CHD patients, particularly those with complex defects who may have unexpected flow dynamics or aortopulmonary or venovenous collaterals, we carefully monitor the bolus tracker and manually trigger the scan acquisition.

\section{Radiation}

The effective radiation dose is affected by $\mathrm{x}$-ray tube potential and current, scan mode, exposure time, $z$-axis coverage, and imaging settings used when bolus tracking. The literature suggests that most congenital CHD scanning can be performed at 70 or $80 \mathrm{kV}[32,57]$. While the use of iterative reconstruction techniques allows lower tube currents to be selected for a given patient size, the clinical question to be addressed should primarily determine tube potential. If distal coronary artery anatomy or other fine anatomic detail is not needed, tube currents can be significantly reduced during both acquisition and monitoring scans. $Z$-axis coverage should also be carefully chosen to cover only the area of interest. An infant's cardiovascular anatomy can usually be imaged in $8 \mathrm{~cm}$, while adolescents and adults may require up to $16 \mathrm{~cm}$ or more.

Scanning throughout the cardiac cycle is less commonly performed, but prospectively or retrospectively gated $\mathrm{CT}$ can be used to assess ventricular function in patients who are unable to undergo cardiac MRI. Although low radiation doses have been reported in dual-source high-pitch scanning, functional assessment with older scanners that have lower temporal resolution often produces undesirably high radiation exposure $[32,95,101,102]$.

\section{Conclusion}

Cardiovascular CT has developed into a key tool among congenital cardiac imaging modalities that complements the roles of echocardiography and cardiac MRI in patients of all ages with cyanotic CHD. It can provide information that informs both preoperative planning and postoperative surveillance and facilitates scanning even in critically ill patients or those with implanted devices. In order to optimize imaging in cyanotic CHD patients, the unique characteristics of each defect and each patient must be carefully considered to develop an individualized protocol for each scan.

\section{Compliance with Ethical Standards}

Conflict of Interest Dr. Einstein receives consultancy fees from GE Healthcare and receives grants from Roche Medical Systems, Canon Medical Systems, and the International Atomic Energy Agency.

All other authors have nothing to disclose.

Human and Animal Rights and Informed Consent This article does not contain any studies with human or animal subjects performed by any of the authors.

Open Access This article is distributed under the terms of the Creative Commons Attribution 4.0 International License (http:// creativecommons.org/licenses/by/4.0/), which permits unrestricted use, distribution, and reproduction in any medium, provided you give appropriate credit to the original author(s) and the source, provide a link to the Creative Commons license, and indicate if changes were made.

\section{References}

Papers of particular interest, published recently, have been highlighted as:

- Of importance

•• Of major importance

1. van der LD, Konings EEM, Slager MA, Witsenburg M, Helbing WA, Takkenberg JJM, et al. Birth prevalence of congenital heart disease worldwide: a systematic review and meta-analysis. J Am Coll Cardiol. 2011;58(21):2241-7. 
2. Hoffman JI, Kaplan S. The incidence of congenital heart disease. J Am Coll Cardiol. 2002;39(12):1890-900.

3. Mitchell SC, Korones SB, Berendes HW. Congenital heart disease in 56,109 births. Incidence and natural history. Circulation. 1971;43(3):323-32.

4. Rao PS. Diagnosis and management of cyanotic congenital heart disease: part I. Indian J Pediatr. 2009;76(1):57-70.

5. Quartermain MD, Pasquali SK, Hill KD, Goldberg DJ, Huhta JC, Jacobs JP, et al. Variation in prenatal diagnosis of congenital heart disease in infants. Pediatrics. 2015;136(2):e378-85.

6. Thangaratinam S, Brown K, Zamora J, Khan KS, Ewer AK. Pulse oximetry screening for critical congenital heart defects in asymptomatic newborn babies: a systematic review and meta-analysis. Lancet. 2012;379(9835):2459-64.

7. Khoshnood B, Lelong N, Houyel L, Thieulin A-C, Jouannic J-M, Magnier S, et al. Prevalence, timing of diagnosis and mortality of newborns with congenital heart defects: a population-based study. Heart. 2012;98(22):1667-73.

8. Ailes EC, Gilboa SM, Honein MA, Oster ME. Estimated number of infants detected and missed by critical congenital heart defect screening. Pediatrics. 2015;135(6):1000-8.

9. Gilboa SM, Devine OJ, Kucik JE, Oster ME, Riehle-Colarusso T, Nembhard WN, et al. Congenital heart defects in the United States: estimating the magnitude of the affected population in 2010. Circulation. 2016;134(2):101-9.

10. Feltes TF, Bacha E, Beekman RH, Cheatham JP, Feinstein JA, Gomes AS, et al. Indications for cardiac catheterization and intervention in pediatric cardiac disease: a scientific statement from the American Heart Association. Circulation. 2011;123(22):2607-52.

11. Yang JC-T, Lin M-T, Jaw F-S, Chen S-J, Wang J-K, Shih TT-F, et al. Trends in the utilization of computed tomography and cardiac catheterization among children with congenital heart disease. $\mathrm{J}$ Formos Med Assoc. 2015;114(11):1061-8.

12. Han BK, Lesser AM, Vezmar M, Rosenthal K, Rutten-Ramos S, Lindberg $\mathrm{J}$, et al. Cardiovascular imaging trends in congenital heart disease: a single center experience. J Cardiovasc Comput Tomogr. 2013;7(6):361-6.

13. Gherardi GG, Iball GR, Darby MJ, Thomson JDR. Cardiac computed tomography and conventional angiography in the diagnosis of congenital cardiac disease in children: recent trends and radiation doses. Cardiol Young. 2011;21(6):616-22.

14. Valente AM, Cook S, Festa P, Ko HH, Krishnamurthy R, Taylor $\mathrm{AM}$, et al. Multimodality imaging guidelines for patients with repaired tetralogy of Fallot: a report from the American Society of Echocardiography: developed in collaboration with the Society for Cardiovascular Magnetic Resonance and the Society for Pediatric Radiology. J Am Soc Echocardiogr. 2014;27(2):111-41.

15. Fratz S, Chung T, Greil GF, Samyn MM, Taylor AM, Valsangiacomo Buechel ER, et al. Guidelines and protocols for cardiovascular magnetic resonance in children and adults with congenital heart disease: SCMR expert consensus group on congenital heart disease. J Cardiovasc Magn Reson. 2013;15(1):51.

16. Riesenkampff E, Messroghli DR, Redington AN, GrosseWortmann L. Myocardial T1 mapping in pediatric and congenital heart disease. Circ Cardiovasc Imaging. 2015;8(2):e002504. https://doi.org/10.1161/CIRCIMAGING.114.002504.

17. Carr D, Brown J, Bydder G, Steiner R, Weinmann H, Speck U, et al. Gadolinium-DTPA as a contrast agent in MRI: initial clinical experience in 20 patients. Am J Roentgenol. 1984;143(2):215-24.

18. Nandalur KR, Dwamena BA, Choudhri AF, Nandalur MR, Carlos RC. Diagnostic performance of stress cardiac magnetic resonance imaging in the detection of coronary artery disease: a meta-analysis. J Am Coll Cardiol. 2007;50(14):1343-53.

19. Voit D, Zhang S, Unterberg-Buchwald C, Sohns JM, Lotz J, Frahm J. Real-time cardiovascular magnetic resonance at $1.5 \mathrm{~T}$ using balanced SSFP and $40 \mathrm{~ms}$ resolution. J Cardiovasc Magn Reson. 2013;15(1):79.

20. Andropoulos DB. Effect of anesthesia on the developing brain: infant and fetus. FDT. 2018;43(1):1-11.

21. Warner DO, Zaccariello MJ, Katusic SK, Schroeder DR, Hanson AC, Schulte PJ, et al. Neuropsychological and behavioral outcomes after exposure of young children to procedures requiring general anesthesia: the Mayo Anesthesia Safety in Kids (MASK) study. Anesthes. 2018;129(1):89-105.

22. Wernovsky G, Newburger J. Neurologic and developmental morbidity in children with complex congenital heart disease. J Pediatr. 2003;142(1):6-8.

23. Ramamoorthy C, Haberkern C, Bhananker S, Domino K, Posner $\mathrm{K}$, Campos J, et al. Anesthesia-related cardiac arrest in children with heart disease. Anesth Analg. 2010;110(5):1376-82.

24. Black S. Anesthesia-related cardiac arrest in children with heart disease: data from the Pediatric Perioperative Cardiac Arrest (POCA) registry. Yearbook Anesthesiol Pain Manag. 2011;2011: 103-5.

25. Olchowy C, Cebulski K, Łasecki M, Chaber R, Olchowy A, Kałwak K, et al. The presence of the gadolinium-based contrast agent depositions in the brain and symptoms of gadolinium neurotoxicity - a systematic review. PLoS One. 2017;12(2): e0171704.

26. Research C for DE and Drug Safety and Availability - FDA Drug Safety Communication: FDA warns that gadolinium-based contrast agents (GBCAs) are retained in the body; requires new class warnings. Available from: https://www.fda.gov/Drugs/ DrugSafety/ucm589213.htm Accessed 15 Jan 2019

27. Ellis AR, Mulvihill D, Bradley SM, Hlavacek AM. Utility of computed tomographic angiography in the pre-operative planning for initial and repeat congenital cardiovascular surgery. Cardiol Young. 2010;20(3):262-8.

28. Hong SH, Goo HW, Maeda E, Choo KS, Tsai I-C. User-friendly vendor-specific guideline for pediatric cardiothoracic computed tomography provided by the Asian Society of Cardiovascular Imaging Congenital Heart Disease Study Group: part 1. Imaging techniques. Korean J Radiol. 2019;20(2):190-204.

29. Hill KD, Frush DP, Han BK, Abbott BG, Armstrong AK, DeKemp RA, et al. Radiation safety in children with congenital and acquired heart disease. JACC Cardiovasc Imaging. 2017;10(7):797-818.

30. Johnson JN, Hornik CP, Li JS, Benjamin DK, Yoshizumi T, Reiman RE, et al. Cumulative radiation exposure and cancer risk estimation in children with heart disease. Circulation. 2014;130(2):161-7.

31. Downing TE, McDonnell A, Zhu X, Dori Y, Gillespie MJ, Rome $\mathrm{JJ}$, et al. Cumulative medical radiation exposure throughout staged palliation of single ventricle congenital heart disease. Pediatr Cardiol. 2015;36(1):190-5.

32.• Rigsby CK, McKenney SE, Hill KD, Chelliah A, Einstein AJ, Han BK, et al. Radiation dose management for pediatric cardiac computed tomography: a report from the Image Gently "Have-AHeart" campaign. Pediatr Radiol. 2018;48(1):5-20. Recent pediatric cardiac CT-specific radiation reduction guidelines from the Image Gently alliance.

33. Ghoshhajra BB, Lee AM, Engel L-C, Celeng C, Kalra MK, Brady $\mathrm{TJ}$, et al. Radiation dose reduction in pediatric cardiac computed tomography: experience from a tertiary medical center. Pediatr Cardiol. 2014;35(1):171-9.

34. Hedgire SS, Baliyan V, Ghoshhajra BB, Kalra MK. Recent advances in cardiac computed tomography dose reduction strategies: a review of scientific evidence and technical developments. J Med Imaging (Bellingham). 2017;4(3):031211. https://doi.org/10. 1117/1.JMI.4.3.031211. 
35. Gao W, Zhong YM, Sun AM, Wang Q, Ouyang RZ, Hu LW, et al. Diagnostic accuracy of sub-mSv prospective ECG-triggering cardiac $\mathrm{CT}$ in young infant with complex congenital heart disease. Int J Card Imaging. 2016;32(6):991-8.

36. Trattner S, Chelliah A, Prinsen P, Ruzal-Shapiro CB, Xu Y, Jambawalikar $\mathrm{S}$, et al. Estimating effective dose of radiation from pediatric cardiac CT angiography using a 64-MDCT scanner: new conversion factors relating dose-length product to effective dose. AJR Am J Roentgenol. 2017;208(3):585-94.

37. Han BK, Casey S, Witt D, Leipsic J, Crean A, Nicol E, et al. Development of a congenital cardiovascular computed tomography imaging registry: rationale and implementation. J Cardiovasc Comput Tomogr. 2018;12(3):263-6. Outlines the development of the first multi-center pediatric and congenital heart disease imaging registry to define use variability and risks of cardiac CT, establish best practice guidelines regarding image quality, radiation dose, and anesthesia use, and prospectively collect multi-institutional data.

38. Friedman BA, Schoepf UJ, Bastarrika GA, Hlavacek AM. Computed tomographic angiography of infants with congenital heart disease receiving extracorporeal membrane oxygenation. Pediatr Cardiol. 2009;30(8):1154-6.

39. Rames JD, Kavarana MN, Schoepf UJ, Hlavacek AM. The utility of computed tomographic angiography in a neonate on extracorporeal membrane oxygenation with extreme cyanosis after Blalock-Taussig shunt. Ann Pediatr Cardiol. 2017;10(2):209-11.

40. Healy F, Hanna BD, Zinman R. Pulmonary complications of congenital heart disease. Paediatr Respir Rev. 2012;13(1):10-5.

41. Silversides GJT, Konen E, Hart MA, Webb GD, Therrien J. Pulmonary thrombosis in adults with Eisenmenger syndrome. J Am Coll Cardiol. 2003;42(11):1982-7.

42. Otton JM, Birbara NS, Hussain T, Greil G, Foley TA, Pather N. 3D printing from cardiovascular CT: a practical guide and review. Cardiovasc Diagn Ther. 2017;7(5):507-26.

43. Farooqi KM, Mahmood F. Innovations in preoperative planning: insights into another dimension using $3 \mathrm{D}$ printing for cardiac disease. J Cardiothorac Vasc Anesth. 2018;32(4):1937-45.

44. Sun Z, Lee S-Y. A systematic review of 3-D printing in cardiovascular and cerebrovascular diseases. Anatol J Cardiol. 2017;17(6):423-35.

45. Fraint H, Chelliah A, Lai W, Bacha E. Use of a 3d printed heart model to plan surgical repair of complex congenital heart disease. J Am Coll Cardiol. 2018;65(10 Supplement):A637.

46. Mai CT, Riehle-Colarusso T, O'Halloran A, Cragan JD, Olney RS, Lin A, et al. Selected birth defects data from populationbased birth defects surveillance programs in the United States, 2005-2009: featuring critical congenital heart defects targeted for pulse oximetry screening. Birth Defects Res A Clin Mol Teratol. 2012;94(12):970-83.

47. Dabizzi RP, Caprioli G, Aiazzi L, Castelli C, Baldrighi G, Parenzan L, et al. Distribution and anomalies of coronary arteries in tetralogy of Fallot. Circulation. 1980;61(1):95-102.

48. Tangcharoen T, Bell A, Hegde S, Hussain T, Beerbaum P, Schaeffter T, et al. Detection of coronary artery anomalies in infants and young children with congenital heart disease by using MR imaging. Radiology. 2011;259(1):240-7.

49. Kalfa DM, Serraf AE, Ly M, Le Bret E, Roussin R, Belli E. Tetralogy of Fallot with an abnormal coronary artery: surgical options and prognostic factors. Eur J Cardiothorac Surg. 2012;42(3):e34-9.

50. Vastel-Amzallag C, Le Bret E, Paul J-F, Lambert V, Rohnean A, El Fassy E, et al. Diagnostic accuracy of dual-source multislice computed tomographic analysis for the preoperative detection of coronary artery anomalies in 100 patients with tetralogy of Fallot. J Thorac Cardiovasc Surg. 2011;142(1):120-6.

51. Goo HW. Coronary artery anomalies on preoperative cardiac CT in children with tetralogy of Fallot or Fallot type of double outlet right ventricle: comparison with surgical findings. Int J Card Imaging. 2018;34(12):1997-2009.

52. Hayabuchi $\mathrm{Y}$, Inoue $\mathrm{M}$, Watanabe $\mathrm{N}$, Sakata $\mathrm{M}$, Nabo $\mathrm{MMH}$, Kitagawa $\mathrm{T}$, et al. Assessment of systemic-pulmonary collateral arteries in children with cyanotic congenital heart disease using multidetector-row computed tomography: comparison with conventional angiography. Int J Cardiol. 2010;138(3):266-71.

53. Meinel FG, Huda W, Schoepf UJ, Rao AG, Cho YJ, Baker GH, et al. Diagnostic accuracy of CT angiography in infants with tetralogy of Fallot with pulmonary atresia and major aortopulmonary collateral arteries. J Cardiovasc Comput Tomogr. 2013;7(6):367-75.

54. Jia Q, Cen J, Li J, Zhuang J, Liu H, Zhang Q, et al. Anatomy of the retro-oesophageal major aortopulmonary collateral arteries in patients with pulmonary atresia with ventricular septal defect: results from preoperative CTA. Eur Radiol. 2018;28(7):3066-74.

55. Ryan JR, Moe TG, Richardson R, Frakes DH, Nigro JJ, Pophal S. A novel approach to neonatal management of tetralogy of Fallot, with pulmonary atresia, and multiple aortopulmonary collaterals. JACC Cardiovasc Imaging. 2015;8(1):103-4.

56. Anwar S, Rockefeller T, Raptis DA, Woodard PK, Eghtesady P. $3 \mathrm{D}$ printing provides a precise approach in the treatment of tetralogy of Fallot, pulmonary atresia with major aortopulmonary collateral arteries. Curr Treat Options Cardiovasc Med. 2018;20(1):5 https://doi.org/10.1007/s11936-018-0594-2

57. Han BK, Lesser JR. CT imaging in congenital heart disease: an approach to imaging and interpreting complex lesions after surgical intervention for tetralogy of Fallot, transposition of the great arteries, and single ventricle heart disease. J Cardiovasc Comput Tomogr. 2013;7(6):338-53.

58. Lapierre C, Dubois J, Rypens F, Raboisson M-J, Déry J. Tetralogy of Fallot: preoperative assessment with MR and CT imaging. Diagn Interv Imaging. 2016;97(5):531-41.

59. Yamasaki Y, Nagao M, Yamamura K, Yonezawa M, Matsuo Y, Kawanami S, et al. Quantitative assessment of right ventricular function and pulmonary regurgitation in surgically repaired tetralogy of Fallot using 256-slice CT: comparison with 3-Tesla MRI. Eur Radiol. 2014;24(12):3289-99.

60. Goo HW. Semiautomatic three-dimensional threshold-based cardiac computed tomography ventricular volumetry in repaired tetralogy of Fallot: comparison with cardiac magnetic resonance imaging. Korean J Radiol. 2019;20(1):102-13.

61. Stout KK, Daniels CJ, Aboulhosn JA, Bozkurt B, Broberg CS, Colman JM, et al. 2018 AHA/ACC guideline for the management of adults with congenital heart disease: a report of the American College of Cardiology/American Heart Association Task Force on Clinical Practice Guidelines. J Am Coll Cardiol. 2018;16:25255.

62. Han BK, Moga FX, Overman D, Carter C, Lesser JR. Diagnostic value of contrast-enhanced multiphase computed tomography for assessment of percutaneous pulmonary valve obstruction. Ann Thorac Surg. 2016;101(4):e115-6.

63. Anderson RH, Weinberg PM. The clinical anatomy of transposition. Cardiol Young. 2005;15(S1):76-87.

64. Goo HW. Identification of coronary artery anatomy on dualsource cardiac computed tomography before arterial switch operation in newborns and young infants: comparison with transthoracic echocardiography. Pediatr Radiol. 2018;48(2):176-85. 
65. Yu F, Lu B, Gao Y, Hou Z, Schoepf UJ, Spearman JV, et al. Congenital anomalies of coronary arteries in complex congenital heart disease: diagnosis and analysis with dual-source CT. J Cardiovasc Comput Tomogr. 2013;7(6):383-90.

66. Odawara Y, Kawamura N, Yamasaki Y, Hashimoto J, Ishikawa S, Honda $\mathrm{H}$. Evaluation of coronary artery variations using dualsource coronary computed tomography angiography in neonates with transposition of the great arteries. Jpn J Radiol. 2019. https:// doi.org/10.1007/s11604-018-00807-x.

67. Ou P, Khraiche D, Celermajer DS, Agnoletti G, Le Quan Sang K$\mathrm{H}$, Thalabard JC, et al. Mechanisms of coronary complications after the arterial switch for transposition of the great arteries. J Thorac Cardiovasc Surg. 2013;145(5):1263-9.

68. Ou P, Celermajer DS, Marini D, Agnoletti G, Vouhé P, Brunelle F, et al. Safety and accuracy of 64-slice computed tomography coronary angiography in children after the arterial switch operation for transposition of the great arteries. JACC Cardiovasc Imaging. 2008;1(3):331-9.

69. Legendre A. Coronary events after arterial switch operation for transposition of the great arteries. Circulation. 2003;108(90101): 186II-190.

70. Szymczyk K, Moll M, Sobczak-Budlewska K, Moll JA, Stefańczyk L, Grzelak P, et al. Usefulness of routine coronary CT angiography in patients with transposition of the great arteries after an arterial switch operation. Pediatr Cardiol. 2018;39(2): $335-46$.

71. Leccisotti L, Bruno I, Stefanelli A, Caldarella C, Giordano A. Transposition of the great arteries: a myocardial perfusion PETCT study. Clin Nucl Med. 2013;38(12):e467-70.

72. Cohen MS, Eidem BW, Cetta F, Fogel MA, Frommelt PC, Ganame J, et al. Multimodality imaging guidelines of patients with transposition of the great arteries: a report from the American Society of Echocardiography developed in collaboration with the Society for Cardiovascular Magnetic Resonance and the Society of Cardiovascular Computed Tomography. J Am Soc Echocardiogr. 2016;29(7):571-621.

73. Moons P, Gewillig M, Sluysmans T, Verhaaren H, Viart P, Massin $\mathrm{M}$, et al. Long term outcome up to 30 years after the Mustard or Senning operation: a nationwide multicentre study in Belgium. Heart. 2004;90(3):307-13.

74. Cook SC, McCarthy M, Daniels CJ, Cheatham JP, Raman SV. Usefulness of multislice computed tomography angiography to evaluate intravascular stents and transcatheter occlusion devices in patients with d-transposition of the great arteries after mustard repair. Am J Cardiol. 2004;94(7):967-9.

75. Sharma R, Bhan A, Juneja R, Sunder Kothari S, Saxena A, Venugopal P. Double switch for congenitally corrected transposition of the great arteries. Eur J Cardiothorac Surg. 1999;15(3): 276-82.

76. Di Donato RM, Troconis CJ, Marino B, Carotti A, Iorio FS, Rossi E, et al. Combined mustard and Rastelli operations. An alternative approach for repair of associated anomalies in congenitally corrected transposition in situs inversus [I,D,D]. J Thorac Cardiovasc Surg. 1992;104(5):1246-8.

77. Hong SH, Kim YM, Lee C-K, Lee C-H, Kim SH, Lee SY. 3D MDCT angiography for the preoperative assessment of truncus arteriosus. Clin Imaging. 2015;39(6):938-44.

78. Sharma A, Priya S, Jagia P. Persistent truncus arteriosus on dual source CT. Jpn J Radiol. 2016;34(7):486-93.

79. Oh KH, Choo KS, Lim SJ, Lee HD, Park JA, Jo MJ, et al. Multidetector CT evaluation of total anomalous pulmonary venous connections: comparison with echocardiography. Pediatr Radiol. 2009;39(9):950-4.

80. Yao Q, Hu X, Pa M, Huang G. Non-ECG-gated MDCTA of infracardiac total anomalous pulmonary venous connection in neonates and young infants. Herz. 2013;38(5):539-43.

81. Deshmukh S, Thompson WR, Zimmerman SL. Total anomalous pulmonary venous connection in a neonate characterised by lowdose, high-pitch cardiac CT. Cardiol Young. 2015;25(06):1197-9.

82. Turkvatan A, Tola HT, Ayyildiz P, Ozturk E, Ergul Y, Guzeltas A. Total anomalous pulmonary venous connection in children: preoperative evaluation with low-dose multidetector computed tomographic angiography. Tex Heart Inst J. 2017;44(2):120-6.

83. Ricci M, Elliott M, Cohen GA, Catalan G, Stark J, de Leval MR, et al. Management of pulmonary venous obstruction after correction of TAPVC: risk factors for adverse outcome. Eur J Cardiothorac Surg. 2003;24(1):28-36.

84. Han BK, Huntley M, Overman D, Witt D, Dassenko D, Garberich RF, et al. Cardiovascular CT for evaluation of single-ventricle heart disease: risks and accuracy compared with interventional findings. Cardiol Young. 2018;28(1):9-20.

85. Han BK, Overman DM, Grant K, Rosenthal K, Rutten-Ramos S, Cook D, et al. Non-sedated, free breathing cardiac CT for evaluation of complex congenital heart disease in neonates. J Cardiovasc Comput Tomogr. 2013;7(6):354-60.

86. Chaosuwannakit N, Makarawate P. Diagnostic accuracy of lowdose dual-source cardiac computed tomography as compared to surgery in univentricular heart patients. J Cardiothorac Surg. 2018;13(1):39. https://doi.org/10.1186/s13019-018-0729-2.

87. Wolla CD, Hlavacek AM, Schoepf UJ, Bucher AM, Chowdhury S. Cardiovascular manifestations of heterotaxy and related situs abnormalities assessed with CT angiography. J Cardiovasc Comput Tomogr. 2013;7(6):408-16.

88. Han BK, Huntley M, Overman D, Witt D, Dassenko D, Garberich RF, et al. Cardiovascular CT for evaluation of single-ventricle heart disease: risks and accuracy compared with interventional findings. Cardiol Young. 2018;28(1):9-20.

89. Han BK, Vezmar M, Lesser JR, Michalak G, Grant K, Dassenko $\mathrm{D}$, et al. Selective use of cardiac computed tomography angiography: an alternative diagnostic modality before second-stage single ventricle palliation. J Thorac Cardiovasc Surg. 2014;148(4):1548 54.

90. Kardos M. Detection of right ventricle thrombosis in patient with Ebstein anomaly of tricuspid valve after Fontan procedure by CT. J Cardiovasc Comput Tomogr. 2014;8(3):248-9.

91. Singh HR, Forbes TJ, Humes RA. CT artifact mimicking pulmonary embolism in a patient with single ventricle. Pediatr Cardiol. 2008;29(1):241-2.

92. Grewal J, Hussein MA, Feldstein J, Kiess M, Ellis J, Human D, et al. Evaluation of silent thrombus after the Fontan operation. Congenit Heart Dis. 2013;8(1):40-7.

93. Prabhu SP, Mahmood S, Sena L, Lee EY. MDCT evaluation of pulmonary embolism in children and young adults following a lateral tunnel Fontan procedure: optimizing contrastenhancement techniques. Pediatr Radiol. 2009;39(9):938-44.

94. Han BK, Rigsby CK, Hlavacek A, Leipsic J, Nicol ED, Siegel MJ, et al. Computed tomography imaging in patients with congenital heart disease part I: rationale and utility. An expert consensus document of the Society of Cardiovascular Computed Tomography (SCCT). J Cardiovasc Comput Tomogr. 2015;9(6): 475-92.

95. Han BK, Rigsby CK, Leipsic J, Bardo D, Abbara S, Ghoshhajra $\mathrm{B}$, et al. Computed tomography imaging in patients with 
congenital heart disease, part 2: technical recommendations. An expert consensus document of the Society of Cardiovascular Computed Tomography (SCCT). J Cardiovasc Comput Tomogr. 2015;9(6):493-513.

96. Raimondi F, Warin-Fresse K. Computed tomography imaging in children with congenital heart disease: indications and radiation dose optimization. Arch Cardiovasc Dis. 2016;109(2):150-7.

97. Pierpont ME, Basson CT, Benson DW, Gelb BD, Giglia TM, Goldmuntz E, et al. Genetic basis for congenital heart defects: current knowledge: a scientific statement from the American Heart Association Congenital Cardiac Defects Committee, Council on Cardiovascular Disease in the Young: endorsed by the American Academy of Pediatrics. Circulation. 2007;115(23): 3015-38.

98. Scholtz J-E, Ghoshhajra B. Advances in cardiac CT contrast injection and acquisition protocols. Cardiovasc Diagn Ther. 2017;7(5):439-51.

99. Dittrich S, Haas NA, Bührer C, Müller C, Dähnert I, Lange PE. Renal impairment in patients with long-standing cyanotic congenital heart disease. Acta Paediatr. 1998;87(9):949-54.
100. Morgan C, Al-Aklabi M, Garcia Guerra G. Chronic kidney disease in congenital heart disease patients: a narrative review of evidence. Can J Kidney Health Dis. 2015;2:27. https://doi.org/ 10.1186/s40697-015-0063-8.

101. Han BK, Hlavacek AM, Kay WA, Pham TDN, Grant K, Garberich RF, et al. Multi-institutional evaluation of the indications and radiation dose of functional cardiovascular computed tomography (CCT) imaging in congenital heart disease. Int $\mathrm{J}$ Cardiovasc Imaging. 2016;32(2):339-46.

102. Le Roy J, Vernhet Kovacsik H, Zarqane H, Vincenti M, Abassi H, Lavastre K, et al. Submillisievert multiphasic coronary computed tomography angiography for pediatric patients with congenital heart diseases. Circ Cardiovasc Imaging. 2019;12(2):e008348. Demonstrated the feasibility of high-quality, low-radiation functional coronary $\mathrm{CT}$ imaging in children with $\mathrm{CHD}$.

Publisher's Note Springer Nature remains neutral with regard to jurisdictional claims in published maps and institutional affiliations. 